\title{
Modeling Aspects for Step and Bus Topologies under Distributed Computing System
}

\author{
Taskeen Zaidi \\ Deptt. of Computer Science \\ Babasaheb Bhimrao Ambedkar University \\ Vidya Vihar, Rae Bareli, Lucknow
}

\author{
Vipin Saxena \\ Professor, Deptt. of Computer Science \\ Babasaheb Bhimrao Ambedkar University \\ Vidya Vihar, Rae Bareli Road, Lucknow
}

\begin{abstract}
The distributed computing approach has the several advantages over the old centralized computing approach like reduction of execution time and low cost involvement. Many researchers have applied the modeling concepts for the distributed computing system. The most popular approach is the object-oriented modeling also known as Unified Modeling Language (UML) approach which is a platform independent to any programming language. In the present work, Unified Modeling Language (UML) is used for the various aspects used in the distributed computing system. This paper consists of the modeling of newly developed step topology and compared with the bus topology under distributed computing system. UML activity diagrams are designed for these topologies and the diameters of bus and step topology are also computed by varying the nodes through hope count and represented in the form of table and graph.
\end{abstract}

\section{Keywords}

Distributed computing system, UML, Activity diagram, Step topology, Diameter.

\section{INTRODUCTION}

Topology plays an important role for static interconnection of the computer systems. In the literature, many topologies appeared but some of them like star, bus are frequently used by the network administrator for establishment of the computer centre. In this connection, an important reference Tanenbaum [1] has written a famous book on computer networks which describes the generation of computer networks including network topologies, adhoc networks, wireless networks etc. Since, many of the software industries are using the concept of the distributed computing systems for execution of the tasks on the local area network which are well described by Frouzan [2] and it consists of detailed processing of networks, structures, models, classification and categories of networks. The distributed computing algorithms for execution of the processes are available in Milenkovic [3] who has also explained security threats caused by users and providers on the network. In the current scenario and faster transfer of the data, dynamic connections are also used by the network administrators which are based on the various types of topologies like hyper cube, digital bus, etc. and these are available in Hwang [4]. If one is transferring the data from one machine to the another machine, then the architecture of the computer systems play an important role for transferring of the data and these are well described by Mano[5]. Patterson et al. [6] has described the architectures and recent technologies used in the computers. Sharing of resources, data, files, audios, videos and various information in distributed environment as well as mutual exclusion of tasks for static connection are available in[7-8]
On the other hand, software and hardware architecture research problems can be solved through the modeling language. Due to evolution of the object-oriented technologies, a well known modeling language called as Unified Modeling Language [UML] is used by the various researchers and this modeling language has been created by the OMG group [9-10]. This language is also used for computing the various performance metrics to create the applications for parallel and distributed systems which have been proposed by [11-12]. Booch [13] has described the various stages of the development of the UML and currently version of the UML is used by the various researchers is 2.0. The use of UML for solving the research problem, how to draw read and create projects to make it effective are well explained in [14]. The various prospects of UML to create effective projects are illustrated by Gomaa [15]. Recently, Saxena and Zaidi [16] have proposed a step topology for static interconnection of computer system under distributed environment. In India, distributed computing approach is used to establish the National Knowledge Network (NKN) project with main objective to connect the higher learning Institutions for sharing the knowledge. Recently, Saxena and Zaidi [17] have studies a case study for computing the \% utilization of NKN alongwith the advantages and difficulties faced under this project. Saxena et al. [18] have also measured the performance of processors for different object-oriented software systems under distributed environment. UML modeling of a protocol for establishing the mutual exclusion in the distributed computing system has been done by Saxena and Arora [19]. Ladder topology which is used for the circuit designing and later on performance of this topology is evaluated by the authors [20-21]. Performance of various static and network topologies have been computed by Saxena and Raj [22-23]. Saxena and Zaidi[24] has computed the performance of Endian operating system in which user's identification has been monitored by internet protocol (version4) and users are connected under static step topology.

On the basis of above, the present work emphasized on the modeling aspects for the step topology and it is compared with the similar kind of famous bus topology. The UML activity and class diagrams are designed for these two topologies used for the static interconnection under the distributed computing environment. Before this, a process id is created for the execution of the processes on the static interconnection and later on a comparison between bus and step topologies are also explained in the form of table and graph.

\section{BACKGROUND}

\subsection{A Distributed System}

Distributed system is a collection of various devices which are communicated to each other by message passing techniques. Devices like laptops, ipads, pdas, etc are connected under 
distributed computing system. Distributed computing reduces cost of infrastructure, allows resources sharing, files sharing, audio sharing, video sharing, data sharing, etc. In India interconnections of these systems is called as National Knowledge Network (NKN) and it is implemented under Next Generation Network (NGN) services. An interconnection of devices under distributed system is shown below in fig. 1 .

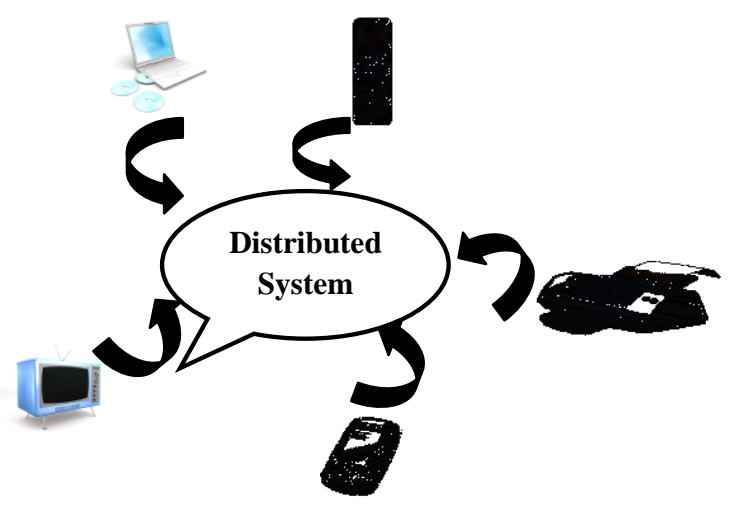

Fig. 1: A distributed system

\subsection{Process}

A process is defined as subprograms, subtasks, macros, subroutines, sub processes which are executed by the processor. PEC i.e. Process Execution Controller is attached to the processes to execute the tasks. The PEC is controlled by the object under object-oriented technology. The class diagram of process is shown below in fig.2, stereotype processing unit is shown in fig. 3 and instances and multiple instances of process are represented in figs.4(a) and(b).

\begin{tabular}{|l|}
\hline \multicolumn{1}{|c|}{$\begin{array}{c}\text { < processing_unit>> } \\
\text { Process }\end{array}$} \\
\hline +Process_id: integer \\
+Process_size: integer \\
+Process_in_time: string \\
+Process_out_time: string \\
+Process_priority: integer \\
+Process_create() \\
+Process_delete()
\end{tabular}

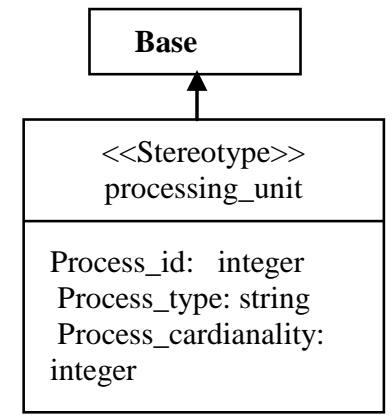

Fig. 2: UML class diagram of process Fig. 3: UML class for processing unit
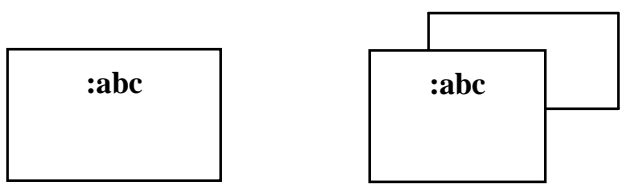

Figs. 4a: Single instance 4b: Multiple instances

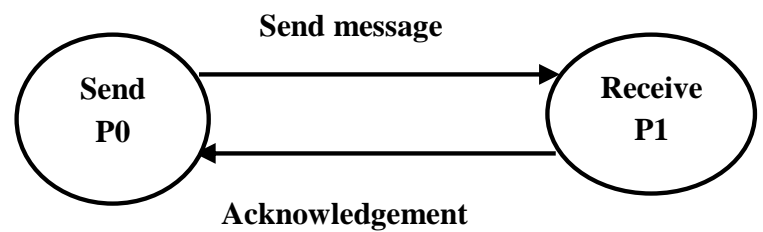

Fig. 5: SEND and RECEIVE operations in process

\section{Communication}

With the help of SEND operation process P0 can send message/request to process $\mathrm{P} 1$ and the request is received with RECEIVE operation by process $\mathrm{P} 1$ as shown in fig. 5 .

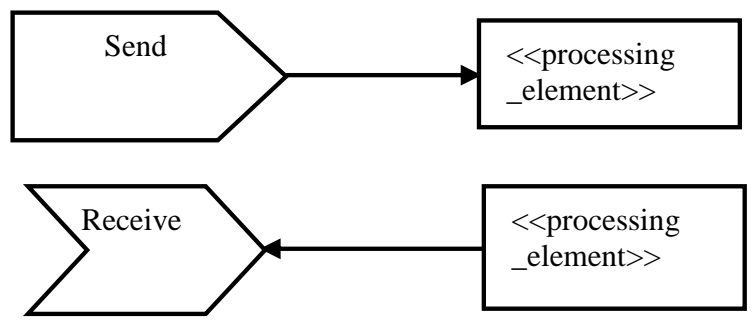

Fig. 6: UML class diagrams of SEND and RECEIVE message

The sending and receiving of messages through UML from processing unit are shown in the fig. 6 .

\subsection{Topologies}

Various kinds of static and dynamic topologies have been proposed by researchers from time to time to connect computers under distributed computing environment. Various types of static topologies like star, tree, ring, mesh etc as well as dynamic topologies like fat tree, barrel shifter, systolic array came into existence. Authors have proposed a new static step topology [23] for interconnection of various computers under distributed computing environment. $\mathrm{N}$ number of computers can be connected by varying the cable length as represented in fig. 7(a).

\subsection{A PEC Representation of Static Network Topologies}

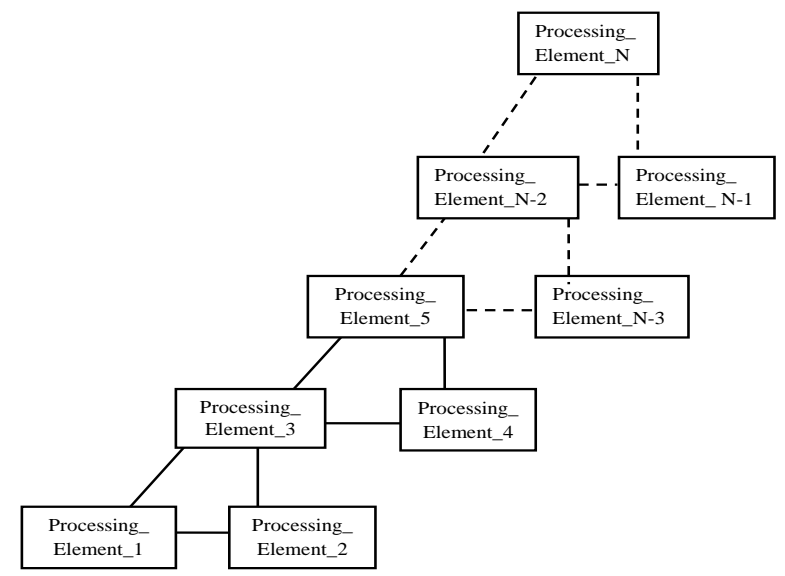

Fig. 7a: A PEC representation of step topology 


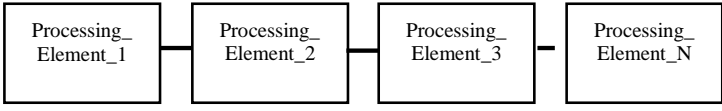

Fig. 7b: A PEC representation of bus topology

In step topology, PEC executes the process on its own node or on next neighbouring node through message passing techniques. $\mathrm{N}$ numbers of computer systems are connected in static step topology under distributed environment as represented in fig. 7(a). While on the other hand, in bus topology, processes are shared on a single communication line as represented in fig. 7(b).

\section{MODELING ASPECTS OF TOPLOGOIES}

A well known Unified Modeling Language is used to represent step and bus topologies by the class and activity diagram. The activities may be the categories of fork and join the two or more than two activities as represented in figs. 8(a) and $8(\mathrm{~b})$, respectively. The activity diagram shows the functioning of the topology and for bus topology, it is represented in fig. 9. In this figure, let $\mathrm{N}$ processes are to be executed under distributed computing system through bus topology. The activity of first process is to be executed under PEC and remaining processes $\mathrm{P}_{2}, \ldots \ldots \mathrm{P}_{\mathrm{N}}$ have to wait for the activities and in the second phase the activity of $\mathrm{P}_{2}$ is performed and in the same manner all the processes are to perform their activities which is in the form of execution of process, sharing of files, etc.

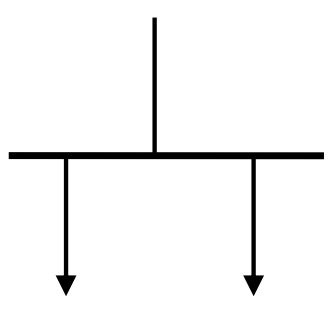

Fig. 8a Process fork

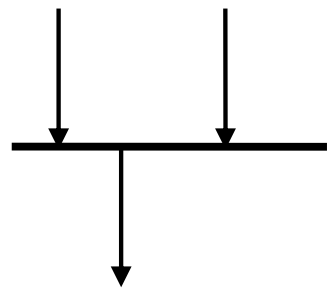

Fig. 8b Process join

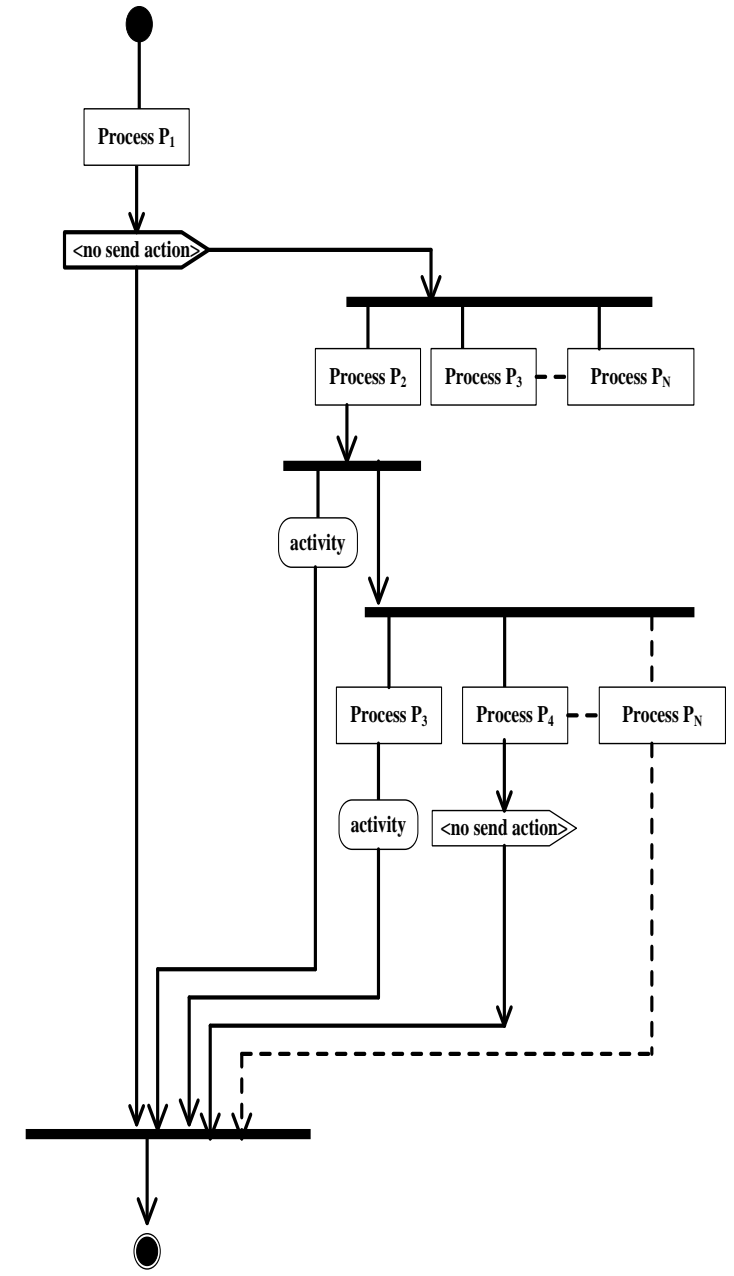

Fig. 9:UML activity diagram for bus topology

In the similar manner, the activity diagram for new kind of step topology is designed and represented in the fig. 10. It is designed in such a way that a first process $\mathrm{P}_{1}$ will perform its activities then only $\mathrm{P}_{2}$ and $\mathrm{P}_{3}$ will wait and remaining processes shall be in queue then in step topology if link between two nodes i.e. Processing_Element1 and Processing_Element2 fails then in such case Processing_Element1 can communicated with Processing_Element 3 by using the alternate path which is not available in the bus topology. In this manner all the processes $\mathrm{P}_{2}, \mathrm{P}_{3} \ldots \ldots . . \mathrm{P}_{\mathrm{N}}$ shall be executed and represented in the following figure. 


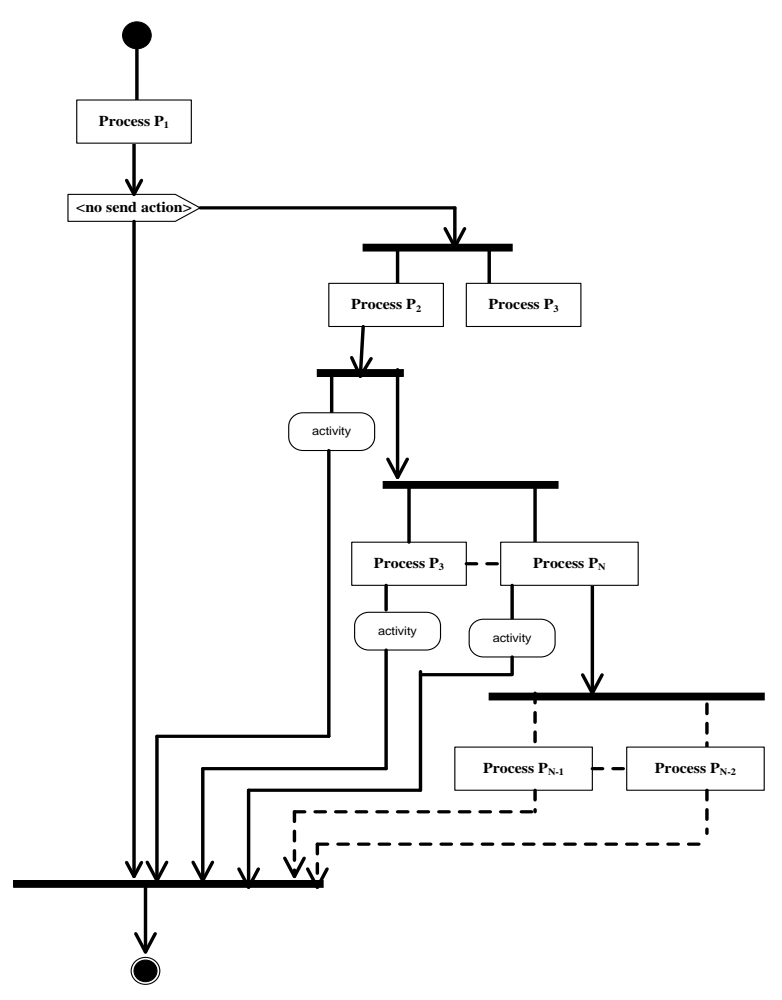

Fig. 10b:UML activity diagram for bus topology

\section{Comparison between Bus and Step Topologies}

\begin{tabular}{|l|l|l|}
\hline Number of Nodes & $\begin{array}{l}\text { Diameter } \\
\text { (Bus Topology) }\end{array}$ & $\begin{array}{l}\text { Diameter } \\
\text { (Step Topology) }\end{array}$ \\
\hline 2 & 1 & 1 \\
\hline 3 & 2 & 2 \\
\hline 4 & 3 & 3 \\
\hline 5 & 4 & 4 \\
\hline 6 & 5 & 5 \\
\hline 7 & 6 & 6 \\
\hline 8 & 7 & 7 \\
\hline 9 & 8 & 8 \\
\hline 10 & 9 & 9 \\
\hline
\end{tabular}

From the figs. 7(a) and 7(b), the diameters of bus and step topologies are computed and shown below in the following table it is observed that the diameter of step topology is same as the bus topology but step topology has the advantage that if link between two nodes fail then it will follow the alternate route to reach to the third node which is not supported by the bus topology. It is observed that as the nodes are increasing, the diameter is also increasing in both the cases and general formula for the diameter is $\mathrm{N}-1$, where is the numbers of nodes available on the network connected wither through bus or step topology. The graphical representation of dfata is also shown below in fig. 11.

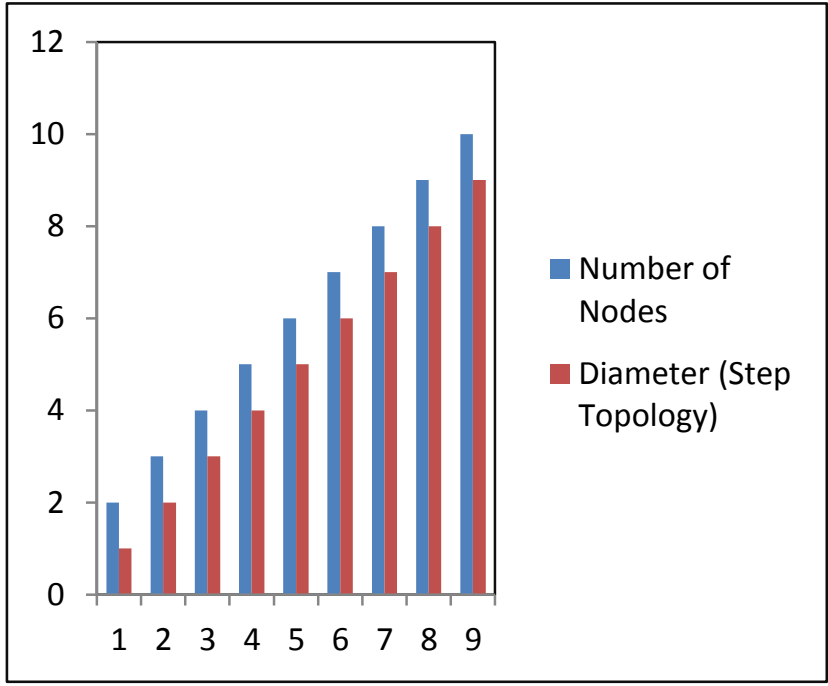

Fig.11 Representation of diameter for step topology

\section{CONCLUSIONS}

From the above work, it is concluded that the UML is a powerful modeling language to represent the software and hardware architecture research problems. In the above work, the activities of a newly developed step topology are demonstrated and compared with the help of bus topology. The execution of the process is done in the processing unit which is fully responsible for execution of the processes and these are demonstrated with the help of UML class diagrams for the step and bus topologies. The comparison between step and bus topology is also demonstrated and observed that if the middle node failures between $N_{1}$ and $N_{3}$ i.e. $N_{2}$ then in the step topology the data is transferred from $\mathrm{N}_{1}$ to $\mathrm{N}_{3}$ as this link does not exist in the bus topology although the diameter will be same in both the cases.

\section{REFERENCES}

[1] Tanenbaum A. S., "Computer Networks", Prentice Hall, 2010.

[2] Frouzen, B. A., "Data Communications and Networks", Tata McGraw Hill, 2006.

[3] Milenkovic, M., "Operating Systems:Concepts and Design”, Tata McGraw Hill, 1987.

[4] Hwang, K., "Advanced Computer Architecture: Parallelism, Scalability, Programmability”, Fourteen reprint, Tata McGraw Hill Edition, 2007, ISBN-0-07, 053070 .

[5] Mano Morris, M., "Computer System Architecture", Third Edition, Prentice Hall of India Pvt Ltd. ISBN-97881-203-0855-8, 2007.

[6] Patterson, A. David and Hennessy, L. John, "Computer Organization and Design: The Hardware/Software Interface”, Morgan Kaufmann Publishers Elsevier Inc., 2005.

[7] Siberschatz, A. and Galvin, P.B. "Operating Systems Concepts", $5^{\text {th }}$ edition, John Wiley \& Sons, Inc, 2000. 
[8] Silberschatz,A. and Peterson, J.L., "Operating System Concepts", Addison Wesley, 1988, Alternate Edition, 1988.

[9] OMG, "Unified Modeling Language Specification". Available online via http://www.omg.org, 2001.

[10] OMG, "Unified Modeling Language (UML)-Version 1.5", OMG document formal/2003-3-01, Needham MA, 2003.

[11] Pllana, S. and Fahringer, T., "On Customizing the UML for Modeling Performance Oriented Applications". In $<<$ UML $>>$, Model Engineering Concepts and Tools, Springer-Verlag., Dresden, Germany, 2002.

[12] Pllana, S.and Fahringer, T., "UML Based Modeling of Performance Oriented Parallel and Distributed Applications", Winter Simulation Conference, 2002.

[13] Booch, G., Rambaugh, J., and Jacobson, I., "The Unified Modeling Language User Guide", Twelfth Indian Reprint Pearson Education, 2004.

[14] Roff, T., “UML:A Beginner's Guide”, Tata McGrawHill Edition. Fifth Reprint, 2006.

[15] Gomaa, H., "Designing Concurrent, Distributed and Real Time Applications with UML", Proceedings of the 23rd International Conference on Software Engineering (ICSE'01), IEEE Computer Society, 2001.

[16] Saxena, V. and Zaidi, T., "Step Topology for Static Interconnection of Computer Systems under Distributed Environment", $3^{\text {rd }}$ World Conference on Information Technology organized by University of Bercelona, Nov. 14-17, 2012.

[17] Saxena, V. and Zaidi , T., "National knowledge Network versus Information Communication Technology", In Proceeding of University Department of Mathematics, B.R.A. Bihar University,Muzzafarfur,India,11-12 Feb.,2012.
[18] Saxena, V., Arora, D. and Ahmad, S., "ObjectOriented Distributed Architecture System through UML", Conference IEEE, International Conference on Advances in Computer Vision and Information Technology, ACVIT-07, ISBN 978-81-89866-74-7, pp.305-310, 2007.

[19] Saxena, V. and Arora, D., 2008 "UML Modeling of a Protocol for Establishing Mutual Exclusion in Distributed Computer System", International Journal of Computer Science and Network Security, Vol.8, No.6, pp.227-235, 2009.

[20] Shiramizu, N., Masuda, T., Nakamura, T. and Washio, K., "Scalable Transformer Model Based on Ladder Topological Equivalent Circuit for SiRFICs", doi: 10.1109/SMIC. 2010.5422954,Letter Symbols for Quantities, ANSI Standard Y10.5-1968, 2010.

[21] Koohazadek, M. and Tamijani, A., "Miniaturized Transmission Lines Based on Hybrid Lattice-Ladder Topology, IEEE Transactions on Microwave Theory and Techniques, Vol.58 (4), 949-955, 2010.

[22] Saxena, V. and Raj, D., "Local Area Performance using UML", International Journal of Advanced Networking \& Applications,Vol.2, page 614-620, 2010.

[23] Saxena, V. and Raj, D., "Modeling of Staic Network Topologies through UML", International journal of Intelligent Information Processing 2(2) July-December, pp.189-197, 2008.

[24] Saxena, V. and Zaidi, T., "Performane of an Endian Operating System for Step Topology", International Journal of Computer Applications" October 2012. 